\title{
Changes in arterial stiffness and nitric oxide production with Chlorella-derived multicomponent supplementation in middle-aged and older individuals
}

\author{
Takeshi Otsuki, ${ }^{1, *}$ Kazuhiro Shimizu and Seiji Maeda ${ }^{3}$ \\ ${ }^{1}$ Faculty of Sport and Health Sciences, Ryutsu Keizai University, 120 Ryugasaki, Ibaraki 301-8555, Japan \\ ${ }^{2}$ Sports Research \& Development Core and ${ }^{3}$ Faculty of Health and Sport Sciences, University of Tsukuba, 1-1-1 Tennoudai, Tsukuba, Ibaraki 305-8577, Japan
}

(Received 22 July, 2015; Accepted 3 August, 2015; Published online 17 October, 2015)

\begin{abstract}
Chlorella is a unicellular green alga, which contains a variety of nutrients including amino acids, dietary fibers, $n-3$ unsaturated fatty acid, vitamins, and minerals. We previously demonstrated that Chlorella-derived multicomponent supplementation decreases arterial stiffness in young men. However, mechanisms underlying the reduction in arterial stiffness by Chlorella-derived supplementation and the effect in middle-aged and older individuals have remained unexplored. This study tested our hypothesis that Chlorella-derived supplementation improves arterial stiffness via an increase in nitric oxide (NO, a endothelium-derived relaxing factor) production in middle-aged and older individuals. Thirty-two subjects between 45 and 75 years of age assigned to placebo and Chlorella groups in a double-blinded manner and took respective tablets for 4 weeks. Before and after the supplementations, brachial-ankle pulse wave velocity (baPWV, an index of arterial stiffness) and plasma nitrite/nitrate (NOx, end product of NO) concentration were measured. There was no difference in baPWV between before and after the placebo intake, but baPWV decreased after the Chlorella supplementation. Changes in baPWV with the Chlorella supplementation were correlated with those in plasma NOx concentration. We concluded that Chlorella-derived multicomponent supplementation decreases arterial stiffness in middle-aged and older individuals. It may be associated with increase in NO production by vascular endothelium.
\end{abstract}

Key Words: nitrite/nitrate, pulse wave velocity, vascular conductance, vascular endothelium

Chlorella is a unicellular green alga and used in Asian and European countries as a dietary supplement. It contains a variety of nutrients including amino acids, dietary fibers, $n-3$ unsaturated fatty acid, vitamins, and minerals. These nutrients can improve arterial stiffness, an independent risk factor for cardiovascular disease. Indeed, we previously reported that Chlorelladerived multicomponent supplementation decreases arterial stiffness in young individuals. ${ }^{(1)}$ However, mechanisms underlying the effect of Chlorella-derived supplementation have remained unexplored. Multicomponent analysis in the previous study showed that Chlorella-derived tablet contains arginine and antioxidants such as vitamin $\mathrm{C}$ and vitamin E. ${ }^{(1)}$ Arginine can be converted to nitric oxide (NO), an endothelium-derived vasodilatory factor, as supplementation with L-arginine increases NO metabolites, nitrite/nitrate (NOx), in urine. ${ }^{(2)}$ Combined intake of vitamin $\mathrm{C}$ and vitamin $\mathrm{E}$ has been demonstrated to improve flowmediated dilation (FMD), an index of NO production by vascular endothelium. ${ }^{(3,4)}$ NO production by vascular endothelium is an important determinant of arterial stiffness. ${ }^{(5,6)}$ Hence, we hypothesized that Chlorella-derived supplementation improves arterial stiffness via an increase in NO production by vascular endothelium. To test this hypothesis, we investigated effects of Chlorella-derived supplementation on brachial-ankle pulse wave velocity (baPWV, an index of arterial stiffness) and plasma NOx concentration using a double-blinded, placebo-controlled study design. In addition, an effect of Chlorella-derived supplementation on arterial stiffness in middle-aged and older individuals has remained unclear. Hence, we recruited middle-aged and older study participants.

\section{Materials and Methods}

Subjects and experimental design. First, a pre-supplementation testing was performed $(n=34)$. The following day, the participants were assigned into either placebo (age, $63.1 \pm 2.3$ years) or Chlorella (62.2 \pm 1.5 years) group in a double-blinded manner. They took 30 tablets per day ( 15 tablets twice daily, after breakfast and dinner) for four weeks. This dosage is in accordance with the general recommended dosage for Japanese consumers. Compliance with the prescription was good in both placebo $(97.4 \pm 2.1 \%)$ and Chlorella $(98.0 \pm 0.5 \%)$ groups. A post-supplementation testing was done one day after the final tablet intake.

A subject whose lifestyle drastically changed during the supplementation period due to his family matter and a subject whose blood sample volume was insufficient to perform all chemical analyses were excluded from statistical analysis. The remaining 32 individuals between the age of 45 and 75 years (placebo group, 6 men and 9 women; Chlorella group, 7 men and 10 women) were included in the analysis. Two subjects in each group were on antihypertensive medication. A subject in the placebo group and two subjects in the Chlorella group were untreated hypertension [systolic blood pressure (SBP)/diastolic blood pressure (DBP) $\geq 140 / 90 \mathrm{mmHg}$. Two subjects in the placebo group and a subject in the Chlorella group were premenopausal women. There were no subjects on hormone replacement therapy and smokers. The participants were asked not to modify their lifestyle during trial period. Subjects refrained from alcohol consumption and intense physical activity starting on the day before testing, and caffeine consumption on the day of testing.

The present study was approved by the Ethical Committee of the Institute of Health and Sport Sciences of the University of

*To whom correspondence should be addressed. E-mail: takeshi.otsuki17@gmail.com 
Tsukuba. This study conformed to the principles outlined in the Helsinki Declaration. All participants gave their written informed consent before their inclusion in this study.

Placebo and Chlorella tablets. The placebo and Chlorella (SunChlorella A; Sun Chlorella Corp., Kyoto, Japan) tablets used in this study were the same as in our previous studies. ${ }^{(1,7,8)}$ The mass of each tablet was 243 and $200 \mathrm{mg}$, respectively. The main components of the placebo tablet were lactose and colorant. The main ingredient of the Chlorella tablet was dried Chlorella pyrenoidosa powder. The nutritional value per $100 \mathrm{~g}$ of the placebo and Chlorella tablets were, respectively: energy, 406 and $399 \mathrm{kcal}$; water, 3.2 and $5.3 \mathrm{~g}$; protein, 2.0 and $60.8 \mathrm{~g}$; lipid, 5.9 and $9.2 \mathrm{~g}$; sugar, 85.6 and $6.3 \mathrm{~g}$; dietary fiber, 1.1 and $11.9 \mathrm{~g}$; and ash, 2.2 and $6.5 \mathrm{~g}$.

Blood biochemistry. Blood samples were collected from the antecubital vein by venipuncture in the morning after an overnight fast. Each blood sample was centrifuged at 2,235 $\mathrm{g}$ for $10 \mathrm{~min}$ at $18^{\circ} \mathrm{C}$. The plasma was stored at $-80^{\circ} \mathrm{C}$ until assay. Plasma concentration of NOx was determined by Griess method as previously described. ${ }^{(9)}$ Serum concentrations of cholesterol, triglycerides, and insulin and plasma levels of glucose and haemoglobin A1c were determined by using the standard techniques. ${ }^{(10)}$

Forearm vascular conductance. Forearm blood flow (BF) at supine position was measured by venous occlusion plethysmography using a mercury-filled strain gauge (EC6; Hokanson, Bellevue). A strain gauge was attached to the widest part of the forearm which was slightly elevated above the level of the right atrium. A wrist cuff was inflated to $50 \mathrm{mmHg}$ above SBP to exclude hand circulation from one minute before onset of forearm $\mathrm{BF}$ measurement to end of the measurement. Upper-arm cuff was rapidly inflated to $40-50 \mathrm{mmHg}$ for $7 \mathrm{~s}$ during a $15 \mathrm{~s}$ cycle to occlude venous outflow from the arm (E20, Hokanson). Forearm $\mathrm{BF}$ was calculated as percent change in forearm volume during the occlusion phase. Eight-cycle measurements were continuously performed and the mean value was calculated. Mean blood pressure (MBP) in contralateral arm was measured two times during the BF measurements by oscillometry (DINAMAP Pro Care 300; GE Healthcare, Buckinghamshire, UK). Forearm vascular conductance (VC) was calculated as the BF divided by the MBP. In our laboratory, the representative day-to-day coefficient of variation for forearm VC was $10.5 \pm 1.1 \%$.

baPWV. baPWV was measured as in our previous study. ${ }^{(11,12)}$ Briefly, brachial and post-tibial artery pressure waveforms were obtained simultaneously by cuffs connected to an air- plethysmographic sensor (formPWV/ABI; Omron Colin, Tokyo, Japan). The distance traveled by the pulse wave from the heart to the brachial recording site and that to the post-tibial recording site were calculated based on each subject's height. The time from when the pulse waves reach the brachial recording site to when they reach the post-tibial recording site was determined based on the time delay between the brachial and post-tibial 'foot' waveforms by the software of the device. baPWV was calculated as the travel distance divided by the travel time. In our laboratory, the representative day-to-day coefficient of variation for baPWV was $2.2 \pm 1.3 \%{ }^{(12)}$ At the time of baPWV measurement, brachial SBP, MBP and DBP were measured using oscillometry. Simultaneously, heart rate (HR) was calculated from an electrocardiogram (formPWV/ABI, Omron Colin).

Statistical analysis. Results are given as means \pm standard errors. $P$ values $<0.05$ were considered statistically significant. Intergroup differences in patient compliance and measures before the supplementation were tested using an unpaired $t$ test. The effects of placebo and Chlorella supplementation were assessed by a repeated-measure two-way analysis of variance. If a significant $F$ value was found, a post hoc Fisher's protected least significant differences test was performed.

\section{Results}

There were no differences between the placebo and Chlorella groups in body weight, body mass index, and blood biochemistry before the tablet intake (Table 1). There were no interactions between group and supplementation period in these measures.

No intergroup differences were observed in blood pressure, HR and forearm BF at the pre-intake testing (Table 2). There were no interactions between group and supplementation period in blood pressure and forearm BF. An interaction was identified in HR, but difference between the pre- and post-intake testing was not significant in HR of the placebo or Chlorella group. An intergroup difference in forearm $\mathrm{VC}$ at the pre-intake testing was not observed (Fig. 1). An interaction between group and supplementation period was identified in forearm VC. A change in forearm VC was close to statistical significance with the Chlorella supplementation, but not with the placebo supplementation.

baPWV before the intake period was similar between the placebo and Chlorella groups (Fig. 2). An interaction between group and supplementation period was identified in baPWV. baPWV decreased after the Chlorella supplementation than at

Table 1. Body weight, body mass index, and blood biochemistry before and after supplementation with placebo and Chlorella tablets

\begin{tabular}{llccc}
\hline & & Before & After & Interaction \\
\hline Body weight $(\mathrm{kg})$ & Placebo & $58.2 \pm 2.8$ & $58.4 \pm 2.8$ & $F=0.7$ \\
& Chlorella & $60.1 \pm 2.0$ & $60.5 \pm 1.9$ & $p=0.40$ \\
Body mass index $\left(\mathrm{kg} / \mathrm{m}^{2}\right)$ & Placebo & $22.8 \pm 0.8$ & $22.9 \pm 0.8$ & $F=0.6$ \\
& Chlorella & $23.6 \pm 0.6$ & $23.8 \pm 0.5$ & $p=0.43$ \\
LDL cholesterol (mg/dl) & Placebo & $122 \pm 7$ & $128 \pm 8$ & $F=3.4$ \\
& Chlorella & $124 \pm 4$ & $120 \pm 5$ & $p=0.07$ \\
HDL cholesterol (mg/dl) & Placebo & $72 \pm 6$ & $74 \pm 6$ & $F=0.8$ \\
& Chlorella & $75 \pm 5$ & $74 \pm 5$ & $p=0.36$ \\
Triglycerides (mg/dl) & Placebo & $85 \pm 11$ & $95 \pm 14$ & $F=0.3$ \\
& Chlorella & $90 \pm 10$ & $106 \pm 13$ & $p=0.61$ \\
Glucose (mg/dl) & Placebo & $94 \pm 2$ & $93 \pm 4$ & $F=0.4$ \\
& Chlorella & $98 \pm 2$ & $99 \pm 4$ & $p=0.55$ \\
Hemoglobin A1c (\%) & Placebo & $6.0 \pm 0.1$ & $6.0 \pm 0.1$ & $F=0.1$ \\
& Chlorella & $6.2 \pm 0.1$ & $6.2 \pm 0.1$ & $p=0.77$ \\
Insulin ( $\mu \mathrm{U} / \mathrm{dl})$ & Placebo & $5.4 \pm 0.9$ & $6.0 \pm 1.0$ & $F=0.7$ \\
& Chlorella & $5.4 \pm 0.8$ & $7.5 \pm 1.6$ & $p=0.40$ \\
\hline
\end{tabular}

Values are means $\pm \mathrm{SE}$. 
Table 2. Blood pressure, heart rate, and forearm blood flow before and after supplementation with placebo and Chlorella tablets

\begin{tabular}{llccc}
\hline & & Before & After & Interaction \\
\hline Systolic blood pressure (mmHg) & Placebo & $119 \pm 4$ & $121 \pm 4$ & $F=2.9$ \\
& Chlorella & $125 \pm 4$ & $122 \pm 4$ & $p=0.10$ \\
Mean blood pressure (mmHg) & Placebo & $91 \pm 3$ & $94 \pm 3$ & $F=3.6$ \\
& Chlorella & $98 \pm 4$ & $95 \pm 3$ & $p=0.06$ \\
Diastolic blood pressure (mmHg) & Placebo & $71 \pm 2$ & $74 \pm 3$ & $F=3.9$ \\
& Chlorella & $76 \pm 2$ & $74 \pm 3$ & $p=0.05$ \\
Heart rate (bpm) & Placebo & $63 \pm 2$ & $60 \pm 1$ & $F=4.2$ \\
Forearm blood flow (ml/dl/min) & Chlorella & $64 \pm 2$ & $65 \pm 2$ & $p=0.04$ \\
& Placebo & $3.7 \pm 0.6$ & $3.2 \pm 0.4$ & $F=3.6$ \\
& Chlorella & $3.4 \pm 0.4$ & $4.2 \pm 0.6$ & $p=0.06$ \\
\hline
\end{tabular}

Values are means $\pm \mathrm{SE}$.

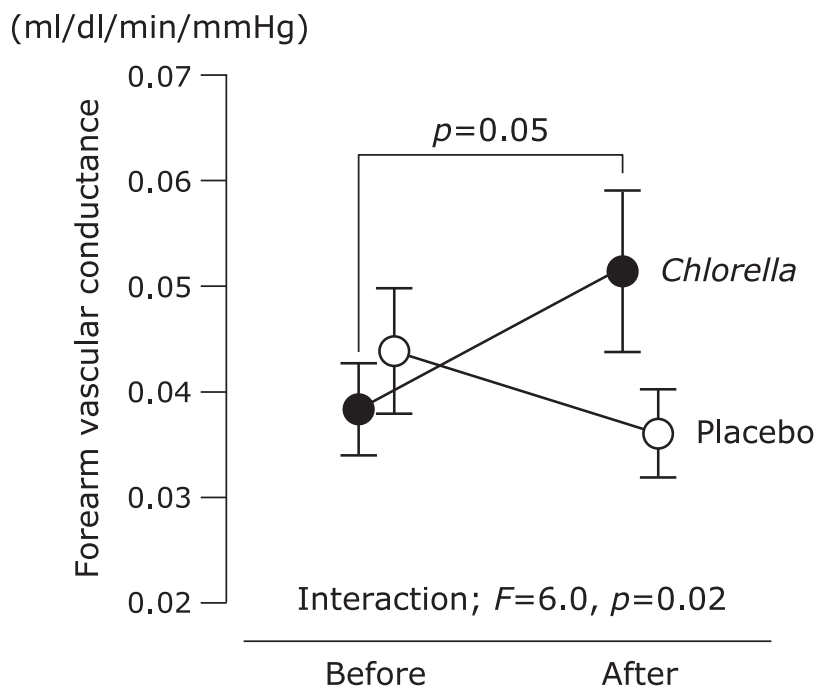

Fig. 1. Forearm vascular conductance before and after supplementation with placebo or Chlorella tablets. Values are means \pm SE.

baseline, whereas the placebo tablets had no effects. There was no difference between the groups in plasma NOx concentration in the pre-supplementation testing (placebo, $43.1 \pm 7.8 \mu \mathrm{mol} / \mathrm{L}$ vs Chlorella, $46.4 \pm 7.2 \mu \mathrm{mol} / \mathrm{L})$. An interaction was not detected in plasma NOx concentration. Plasma NOx concentration increased after the intake period compared to baseline both in the placebo (after, $88.3 \pm 10.8 \mu \mathrm{mol} / \mathrm{L}, p=0.003$ vs before) and Chlorella $(97.9 \pm 20.4 \mu \mathrm{mol} / \mathrm{L}, \quad p=0.01)$ groups. However, changes in plasma NOx concentration with the supplementation period were correlated with changes in baPWV only in the Chlorella group (Fig. 3).

\section{Discussion}

NO is a vasodilatory factor which is produced by vascular endothelium. Local or systemic administrations of a NO synthesis blocker to humans have suggested that NO-related signals participate in regulation of arterial stiffness. ${ }^{(5,6)}$ In this study, effects of Chlorella-derived multicomponent supplementation on baPWV and plasma NOx concentration were investigated by using a double-blinded, placebo-controlled study design. We demonstrated for the first time that Chlorella-derived supplementation improves baPWV in middle-aged and older individuals and that the changes in this measure are correlated with elevations in plasma NOx concentration. Results in subjects without treated

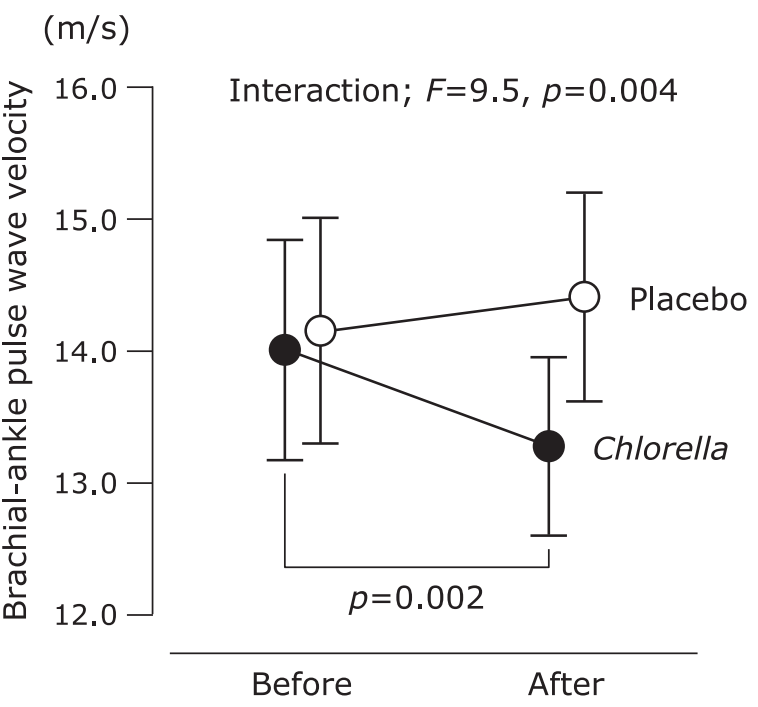

Fig. 2. Brachial-ankle pulse wave velocity before and after supplementation with placebo or Chlorella tablets. Values are means \pm SE.

and untreated hypertension or premenopausal women were similar to results in all subjects (data are not shown). We concluded that Chlorella-derived multicomponent supplementation decreases arterial stiffness in middle-aged and older individuals. It may be associated with increase in NO production by vascular endothelium.

Interpretation of changes in plasma NOx level is not easy. First, many foods in Japanese daily meal contain NOx. ${ }^{(13)}$ Diet before blood sampling possibly affects plasma NOx concentration. ${ }^{(13,14)}$ Intake of NOx from diet might partly increase plasma NOx concentration after the supplementation period. It may be a reason that changes in plasma NOx concentration with the placebo supplementation was not correlated with changes in baPWV. On the other hand, elevations in plasma NOx concentration after the Chlorella supplementation were correlated with decreases in baPWV. There are three subjects with marked elevation of plasma NOx level after the Chlorella supplementation $(>150 \mu \mathrm{mol} / \mathrm{L})$. However, correlation coefficient between changes in plasma NOx concentration and changes in baPWV remained statistically significant in the Chlorella group after these three subjects were excluded (data is not shown). Second, NO is generated from not only endothelial cells but also inflammatory cells. It is possible that Chlorella-derived multicomponent tablet increased the production of NO from inflammatory cells. However, inflammation is positively correlated with arterial stiffness. ${ }^{(15)}$ It is reasonable that 

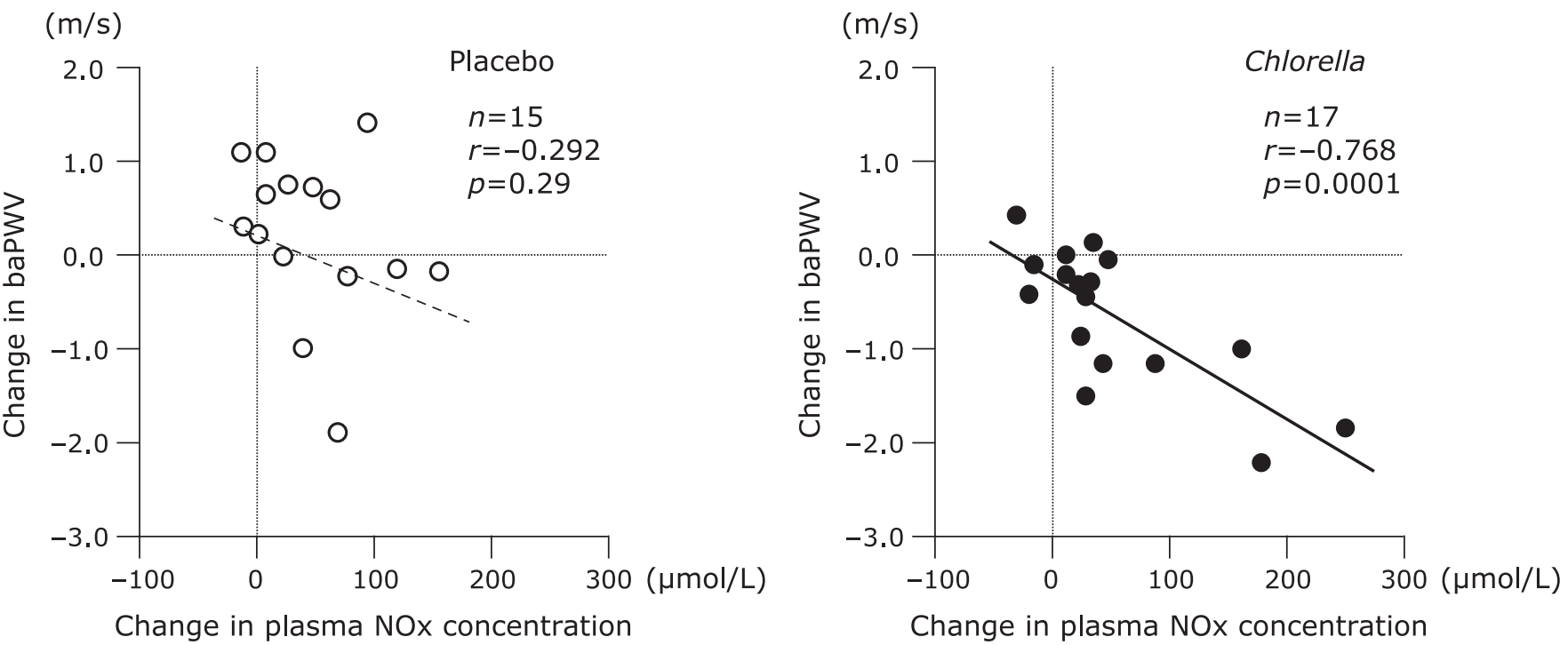

Fig. 3. Relationships between changes in plasma nitrite/nitrate (NOx) concentration and brachial-ankle pulse wave velocity (baPWV) from before to after supplementation with placebo (left panel) or Chlorella (right panel) tablets.

plasma NOx concentrations after the Chlorella supplementation were elevated by vascular endothelium-derived NO rather than inflammatory cell-related NO because the changes in plasma NOx concentrations were inversely correlated with arterial stiffness.

The previous multicomponent analyses of the Chlorella tablet detected arginine (3.16 g/100 g) and antioxidants (e.g., betacarotene, $4.3 \mathrm{mg} / 100 \mathrm{~g}$; vitamin C, $4.0 \mathrm{mg} / 100 \mathrm{~g}$; vitamin $\mathrm{E}$, $2.7 \mathrm{mg} / 100 \mathrm{~g}) .^{(1,8)}$ Bai et al. ${ }^{(16)}$ demonstrated in their meta-analysis that 3-28 days oral supplementation with L-arginine increases FMD, although there was a study which failed to find improvement of FMD by supplementation with L-arginine. ${ }^{(17)}$ The Chlorella-derived supplementation might activate NO production by increasing in blood arginine concentration. Combined ingestion of vitamin $\mathrm{C}$ and vitamin $\mathrm{E}$ have reported to improve FMD in hypertensive middle-aged men and children with hyperlipidemia. ${ }^{(3,4)}$ Additionally, Plantinga et al. ${ }^{(3)}$ found that changes in FMD with antioxidants supplementation were correlated with changes in plasma malondialdehyde level, an index of oxidative stress, although it did not reach statistical significance $(p=0.07)$. It is also possible that the Chlorella-derived supplementation increased NO production through reduction of oxidative stress on vascular endothelium.

Beneficial effects of dietary supplements are more definitively seen when nutrients are taken in combination than in isolation. For example, combination treatment ( $1 \mathrm{~g}$ vitamin $\mathrm{C}$ and $400 \mathrm{IU}$ vitamin $\mathrm{E}$ per day) and the addition of antioxidants (120 mg vitamin $\mathrm{C}, 30 \mathrm{mg}$ vitamin $\mathrm{E}, 6 \mathrm{mg} \beta$-carotene, $100 \mu \mathrm{g}$ selenium, and $20 \mathrm{mg}$ zinc per day) decreased aortic pulse wave velocity $(\mathrm{PWV}){ }^{(3,18)}$ whereas supplementation with vitamin $\mathrm{C}$ alone (500 mg daily) and vitamin $\mathrm{E}$ alone (400 IU daily) did not decrease aortic PWV. ${ }^{(19,20)}$ Strong point of the Chlorella-derived tablet is including various nutrients, even though the dose of each nutrient was small. In addition to arginine and antioxidants, this tablet includes potassium $(970 \mathrm{mg} / 100 \mathrm{~g}){ }^{(1,8)}$ which can affect forearm VC by decreasing contractility of vascular smooth muscle cells via sodium excretion. ${ }^{(21-23)}$ Again, this tablet includes calcium $(330 \mathrm{mg} / 100 \mathrm{~g})$, magnesium $(370 \mathrm{mg} / 100 \mathrm{~g})$, dietary fiber $(11.9 \mathrm{~g} / 100 \mathrm{~g})$ and protein $(60.8 \mathrm{~g} / 100 \mathrm{~g})$;(1,8) $^{(1)}$ these nutrients also have preventive or therapeutic effects on hypertension. ${ }^{(24-26)}$ In this study, an interaction between the groups and the supplementation period in forearm VC was identified and those in MBP and DBP were close to statistical significance. Reduction in peripheral VC decreases blood pressure. Indirect mechanisms based on blood pressure changes might be associated with reduction in arterial stiffness after the Chlorella intake as well as a direct mechanism based on NO production. Additionally, subjects in this study possibly had heterogeneous nutritional status. Nutrients, which were associated with changes in arterials stiffness, might depend on nutritional status in each subject. The Chlorelladerived tablet contains not only favorable but also unfavorable nutrients such as phosphorus (unpublished data); high concentrations of serum phosphorus impair endothelial function via increasing oxidative stress and inflammatory response. ${ }^{(27)}$ However, effects of favorable nutrients on arterial stiffness are likely to overcome effects of unfavorable nutrients. Taken together, the wide spectrum of nutrients in the Chlorella-derived supplementation may be associated with decrease in arterial stiffness.

One meta-analysis showed that the relative risk of a cardiovascular disease event increases $12 \%$ per $1.0 \mathrm{~m} / \mathrm{s}$ increase in baPWV. ${ }^{(28)}$ The observed reduction in baPWV between the pre- and post-Chlorella-derived supplementation was $0.6 \mathrm{~m} / \mathrm{s}$. Chlorelladerived multicomponent supplementation may be beneficial as a primary cardiovascular disease prevention strategy.

This study has several limitations. First, plasma NOx concentration was used for index of vascular endothelial function. As previously mentioned, we recognize the difficulty of the estimation of endothelium-derived NO using the plasma. Second, this is a small placebo-controlled study. The results in this study do not conflict with the previous cross over study in young men. ${ }^{(1)}$ However, the presented results warrant to be confirmed by using a more reliable measure of vascular endothelial function such as FMD in larger placebo-controlled study or cross over study.

In conclusion, Chlorella-derived multicomponent supplementation decreases arterial stiffness in middle-aged and older individuals. It may be associated with increase in NO production by vascular endothelium.

\section{Conflict of Interest}

Sun Chlorella Corp. provided funding for the study and supplied the test supplements. TO received speaker's honorarium from Sun Chlorella Corp. KS and SM have no competing interests. 


\section{Abbreviations}

baPWV brachial-ankle pulse wave velocity

BF blood flow

DBP diastolic blood pressure

FMD flow-mediated dilation

HR heart rate
MBP

NO

NOx

PWV

SBP

$\mathrm{VC}$ mean blood pressure

nitric oxide

nitrite/nitrate

pulse wave velocity

systolic blood pressure

vascular conductance

\section{References}

1 Otsuki T, Shimizu K, Iemitsu M, Kono I. Multicomponent supplement containing Chlorella decreases arterial stiffness in healthy young men. J Clin Biochem Nutr 2013; 53: 166-169.

2 Hishikawa K, Nakaki T, Tsuda M, et al. Effect of systemic L-arginine administration on hemodynamics and nitric oxide release in man. Jpn Heart J 1992; 33: 41-48.

3 Plantinga Y, Ghiadoni L, Magagna A, et al. Supplementation with vitamins $\mathrm{C}$ and $\mathrm{E}$ improves arterial stiffness and endothelial function in essential hypertensive patients. Am J Hypertens 2007; 20: 392-397.

4 Engler MM, Engler MB, Malloy MJ, et al. Antioxidant vitamins C and E improve endothelial function in children with hyperlipidemia: Endothelial Assessment of Risk from Lipids in Youth (EARLY) Trial. Circulation 2003; 108: 1059-1063.

5 Kinlay S, Creager MA, Fukumoto M, et al. Endothelium-derived nitric oxide regulates arterial elasticity in human arteries in vivo. Hypertension 2001; 38: 1049-1053.

6 Sugawara J, Komine H, Hayashi K, et al. Effect of systemic nitric oxide synthase inhibition on arterial stiffness in humans. Hypertens Res 2007; 30: 411-415.

7 Otsuki T, Shimizu K, Iemitsu M, Kono I. Salivary secretory immunoglobulin A secretion increases after 4-weeks ingestion of Chlorella-derived multicomponent supplement in humans: a randomized cross over study. Nutr $J$ 2011; 10: 91 .

8 Otsuki T, Shimizu K, Iemitsu M, Kono I. Chlorella intake attenuates reduced salivary SIgA secretion in kendo training camp participants. Nutr J 2012; 11: 103.

9 Green LC, Wagner DA, Glogowski J, Skipper PL, Wishnok JS, Tannenbaum SR. Analysis of nitrate, nitrite, and [15N]nitrate in biological fluids. Anal Biochem 1982; 126: 131-138.

10 Otsuki T, Maeda S, Mukai J, Ohki M, Nakanishi M, Yoshikawa T. Association between plasma sLOX-1 concentration and arterial stiffness in middleaged and older individuals. J Clin Biochem Nutr 2015; 57: 151-155.

11 Otsuki T, Ohashi C. Participation in physical activity and arterial stiffness in males with autism spectrum disorder. Artery Res 2014; 8: 110-114.

12 Otsuki T, Takanami Y, Aoi W, Kawai Y, Ichikawa H, Yoshikawa T. Arterial stiffness acutely decreases after whole-body vibration in humans. Acta Physiol (Oxf) 2008; 194: 189-194.

13 Himeno $\mathrm{M}$, Ishibashi T, Nakano S, et al. A practical procedure for achieving a steady state of NOx concentration in plasma: with special reference to the NOx content of Japanese daily food. Tohoku J Exp Med 2003; 199: 95-110.

14 Wang J, Brown MA, Tam SH, Chan MC, Whitworth JA. Effects of diet on measurement of nitric oxide metabolites. Clin Exp Pharmacol Physiol 1997; 24: $418-420$.

15 Vlachopoulos C, Dima I, Aznaouridis K, et al. Acute systemic inflammation increases arterial stiffness and decreases wave reflections in healthy individuals.
Circulation 2005; 112: 2193-2200.

16 Bai Y, Sun L, Yang T, Sun K, Chen J, Hui R. Increase in fasting vascular endothelial function after short-term oral L-arginine is effective when baseline flow-mediated dilation is low: a meta-analysis of randomized controlled trials. Am J Clin Nutr 2009; 89: 77-84.

17 Adams MR, Forsyth CJ, Jessup W, Robinson J, Celermajer DS. Oral Larginine inhibits platelet aggregation but does not enhance endotheliumdependent dilation in healthy young men. J Am Coll Cardiol 1995; 26: 1054 1061.

18 Zureik M, Galan P, Bertrais S, et al. Effects of long-term daily low-dose supplementation with antioxidant vitamins and minerals on structure and function of large arteries. Arterioscler Thromb Vasc Biol 2004; 24: 14851491.

19 Eskurza I, Monahan KD, Robinson JA, Seals DR. Ascorbic acid does not affect large elastic artery compliance or central blood pressure in young and older men. Am J Physiol Heart Circ Physiol 2004; 286: H1528-H1534.

20 Rasool AH, Rehman A, Wan Yusuf WN, Rahman AR. Vitamin E and its effect on arterial stiffness in postmenopausal women--a randomized controlled trial. Int J Clin Pharmacol Ther 2003; 41: 587-592.

21 Mano M, Sugawara A, Nara Y, et al. Potassium accelerates urinary sodium excretion during salt loading without stimulating atrial natriuretic polypeptide secretion. Clin Exp Pharmacol Physiol 1992; 19: 795-801.

22 Smith SJ, Markandu ND, Sagnella GA, Poston L, Hilton PJ, MacGregor GA. Does potassium lower blood pressure by increasing sodium excretion? A metabolic study in patients with mild to moderate essential hypertension. $J$ Hypertens Suppl 1983; 1: 27-30.

23 Voors AW, Dalferes ER, Jr, Frank GC, Aristimuno GG, Berenson GS Relation between ingested potassium and sodium balance in young Blacks and whites. Am J Clin Nutr 1983; 37: 583-594.

24 Appel LJ, Moore TJ, Obarzanek E, et al. A clinical trial of the effects of dietary patterns on blood pressure. DASH Collaborative Research Group. $N$ Engl J Med 1997; 336: 1117-1124.

25 Appel LJ, Brands MW, Daniels SR, Karanja N, Elmer PJ, Sacks FM; American Heart Association. Dietary approaches to prevent and treat hypertension: a scientific statement from the American Heart Association. Hypertension 2006; 47: 296-308.

26 Savica V, Bellinghieri G, Kopple JD. The effect of nutrition on blood pressure. Annu Rev Nutr 2010; 30: 365-401.

27 Watari E, Taketani Y, Kitamura T, et al. Fluctuating plasma phosphorus level by changes in dietary phosphorus intake induces endothelial dysfunction. J Clin Biochem Nutr 2015; 56: 35-42.

28 Vlachopoulos C, Aznaouridis K, Terentes-Printzios D, Ioakeimidis N, Stefanadis C. Prediction of cardiovascular events and all-cause mortality with brachial-ankle elasticity index: a systematic review and meta-analysis. Hypertension 2012; 60: 556-562. 